\title{
Synthesis of Platinum Nanoparticles by Gamma Co-60 Ray Irradiation Method Using Chitosan as Stabilizer
}

\author{
Thi Kim Lan Nguyen, ${ }^{1}$ Ngoc Duy Nguyen, ${ }^{1}$ Van Phu Dang, ${ }^{1}$ Dinh Tuan Phan $\mathbb{D}^{2}$ \\ Thai Hoa Tran $\left(\mathbb{D},{ }^{3}\right.$ and Quoc Hien Nguyen ${ }^{1}{ }^{1}$ \\ ${ }^{1}$ Research and Development Center for Radiation Technology, Vietnam Atomic Energy Institute, 202A Street 11, \\ Linh Xuan Ward, Thu Duc District, Ho Chi Minh City 700000, Vietnam \\ ${ }^{2}$ Hochiminh City University of Natural Resources and Environment, 263B Le Van Sy Street, Ho Chi Minh City 700000, Vietnam \\ ${ }^{3}$ College of Sciences, Hue University, Hue 530000, Vietnam
}

Correspondence should be addressed to Thai Hoa Tran; trthaihoa@yahoo.com and Quoc Hien Nguyen; hien7240238@yahoo.com

Received 4 January 2019; Accepted 24 March 2019; Published 18 April 2019

Guest Editor: Hien Duy Mai

Copyright (C) 2019 Thi Kim Lan Nguyen et al. This is an open access article distributed under the Creative Commons Attribution License, which permits unrestricted use, distribution, and reproduction in any medium, provided the original work is properly cited.

\begin{abstract}
Platinum nanoparticles were synthesized using the gamma-ray irradiation as a reducing factor and chitosan as a stabilizer. The prepared platinum nanoparticles were characterized using ultraviolet-visible spectroscopy (UV-Vis), transmission electron microscopy (TEM), and Fourier-transform infrared spectroscopy (FT-IR). The saturated conversion dose to reduce the $\mathrm{Pt}^{4+}$ to $\mathrm{Pt}^{0}$ was found to be about $14 \mathrm{kGy}$ for initial $\mathrm{Pt}^{4+}$ concentration of $1 \mathrm{mM}$. The size of the platinum nanoparticles $(1.4-1.6 \mathrm{~nm})$ did almost not change with the increase of chitosan concentration from 0.5 to $2.0 \%$. Thus, gamma Co-60 ray irradiation method is favorable for synthesizing PtNPs with the small sizes.
\end{abstract}

\section{Introduction}

Platinum nanoparticles (PtNPs) have attracted great attention in many fields due to their applications in catalysis [1-7], such as in fuel cell technology [1], exhaust purification [2], automobile [3], and energy storage and sensors [8]. However, the possible applications are related to the size and morphology of PtNPs [1, 3, 8, 9]. PtNPs with small sizes possess a large number of surface atoms that affect its surface activity [8]. So, it is essential to prepare small size of PtNPs. The synthesis of PtNPs has already been studied using different methods such as chemical reduction [8-13], electrochemical deposition [1,8], hydrothermal reduction $[1,3]$, biological synthesis [14], gamma Co-60 ray irradiation $[4,6]$, and polyol method [1]. In comparison with other methods, the gamma Co-60 ray irradiation is considered as an effective method for the synthesis of metal nanoparticles due to several advantages such as (1) the reduction reaction of metal ions performed at ambient condition; (2) the rate of reduction reaction can be properly controlled; (3) the reducing agents uniformly distributed in the solution; and (4) large-scale production can be favorably set up and satisfied with requirements of the clean production process [6]. Stabilizers, such as dextran [3], polyvinyalcohol, polyacrylic acid, and polyacrylate [4], and chitosan [5], and others must be used for the protection of PtNPs from agglomeration. Among stabilizers studied, chitosan (CTS) is widely used in the synthesis of metallic nanoparticles such as gold, platinum, silver, and palladium [10]. The CTS is one of the environmentally friendly polymers that is particularly interesting in the synthesis of metal nanoparticles due to its interactions with metal nanoparticles through both steric and electrostatic effect. The polymer molecules bind with the nanoparticles to prevent leaching from the catalysts. These characteristics make CTS an ideal material as catalystic support $[1,5,11]$. To the best of our knowledge, there has been no research on the synthesis of PtNPs with small size (1-2 nm) and narrow particle size distribution by the 
radiolytic method. In this study, the synthesis of PtNPs by the gamma Co-60 ray irradiation using CTS as a stabilizer was carried out, and the effect of CTS concentration on the size of PtNPs was also investigated.

\section{Materials and Methods}

2.1. Materials and Chemicals. Potassium hexachloroplatinate (IV) $\left(\mathrm{K}_{2} \mathrm{PtCl}_{6}\right)$ was purchased from Merck. CTS with the molecular weight of $\sim 95,000 \mathrm{~g} / \mathrm{mol}$ and deacetylation degree of $\sim 90 \%$ were supplied by a factory in Vung Tau Province, Vietnam. Lactic acid was purchased from Shanghai Chemical Reagent Co., China. Distilled water was used in all experiments.

2.2. Preparation of $\mathrm{Pt}^{4+} / \mathrm{CTS}$ Solution and Gamma-Ray Irradiation. Three-gram CTS was dissolved in $100 \mathrm{ml} \mathrm{lac-}$ tic acid $1.5 \%(\mathrm{v} / \mathrm{v})$ to prepare a $3 \%(\mathrm{w} / \mathrm{v})$ CTS solution. $\mathrm{K}_{2} \mathrm{PtCl}_{6}$ was then dissolved in CTS solutions to prepare $\mathrm{Pt}^{4+}$ / CTS solutions with $1 \mathrm{mM} \mathrm{Pt}^{4+}$ and different CTS concentrations of $0.5,1.0$, and $2.0 \%(\mathrm{w} / \mathrm{v})$. Then, the solutions of $\mathrm{Pt}^{4+} /$ CTS were poured into glass bottles with caper and irradiated on the gamma Co-60 irradiator, STSV Co-60/B (Hungary), at VINAGAMMA Center, Ho Chi Minh City, with doses up to $17.5 \mathrm{kGy}$ (dose rate of $1.25 \mathrm{kGy} / \mathrm{h}$ ) measured by the dichromate dosimetry system [15].

2.3. Characterization of Colloidal PtNPs/CTS Solution. The UV-Vis absorption spectra of PtNPs/CTS solutions were measured on a UV-Vis spectrophotometer model Jasco-V630, Japan. The size and size distribution of the PtNPs were determined from TEM images on a transmission electron microscope model JEM 1010, JEOL, Japan, operating at $80 \mathrm{kV}$ and statistically calculated using Photoshop software [16]. The FT-IR spectra of samples were measured on a FT-IR spectrometer 8400 S, Shimadzu, Japan. For FT-IR measurement, the PtNPs/CTS solutions were dried at $60^{\circ} \mathrm{C}$ in a forced air oven. The dried PtNPs/CTS samples were ground into fine powder. After that, the powder samples of PtNPs/CTS were mixed with $\mathrm{KBr}$ and compressed into pellets with a thickness of $0.5 \mathrm{~mm}$.

\section{Results and Discussion}

The $1 \mathrm{mM} \mathrm{Pt}^{4+} / 1 \%$ CTS solution was irradiated with the different doses of 3.5, 7.0, 10.5, 14.0, and 17.5 kGy did almost not have clearly maximum absorption peak $\left(\lambda_{\max }\right)$ in the wavelength range of the measured UV-Vis spectrum, but the absorbance was increased with the increase of dose up to $14 \mathrm{kGy}$ (Figure 1). After irradiation, the color of the initial colorless $\mathrm{Pt}^{4+} / \mathrm{CTS}$ solutions was converted to brown color, and the higher the absorption dose, the darker the color of PtNPs/CTS solutions obtained. The same pattern of UV-Vis spectrum of PtNPs with no $\lambda_{\max }$ was also reported by Yang et al. [3] using the chemical reduction, and by Remita et al. [4] and Choi et al. [6] using the gamma Co-60 ray irradiation method. However, Gharibshahi et al. [17] recently have reported that the PtNPs $(2.8-4.4 \mathrm{~nm})$ synthesized by the

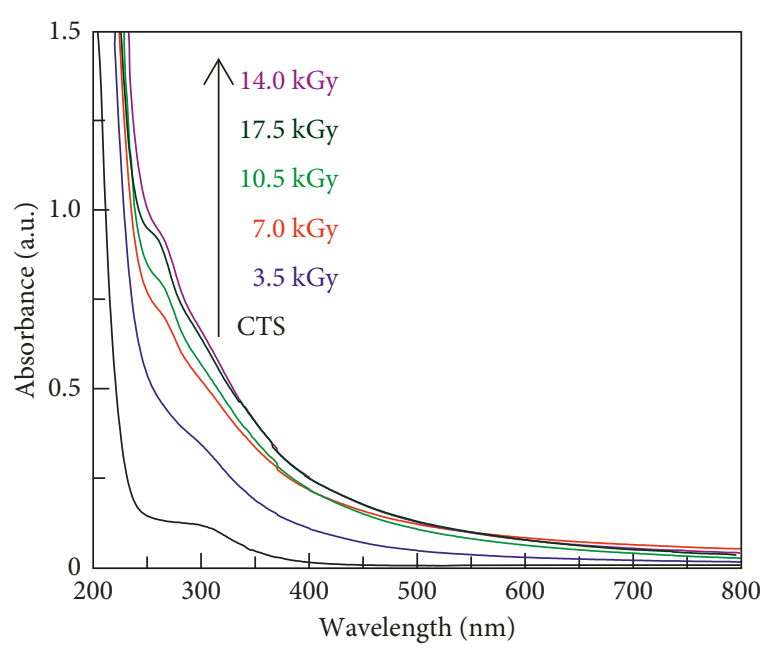

FIgURE 1: UV-Vis spectra of CTS and $\mathrm{Pt}^{4+} / \mathrm{CTS}$ solutions irradiated with different doses.

gamma-ray irradiation of $3.4 \mathrm{mM} \mathrm{Pt}^{4+}$ solution appeared two $\lambda_{\max }$ in the UV-Vis spectrum, particularly the first peak in the range of 209-214 $\mathrm{nm}$ and the second one in the range of 257-262 nm. Furthermore, Cele et al. [18] reported that the PtNPs solutions prepared by the gamma-ray radiolytic method with the concentrations from 1 to $10 \mathrm{mM}$ also had two $\lambda_{\max }$ as in the study of Gharibshahi et al. [17] except for the sample of $1 \mathrm{mM}$ PtNPs without $\lambda_{\max }$ in the UV region due to less Pt atoms interacting with the light.

Figure 1 shows that the CTS solution has a weak peak at $\sim 300 \mathrm{~nm}$. All spectra of PtNPs/CTS solutions also manifested a weak peak at $260-300 \mathrm{~nm}$ with increased absorbance corresponding to the increase of the absorbed dose. This may be due to the formation of carbonyl groups from CTS by chain scission during irradiation [19]. Besides, the absorbance was increased with the increase of dose and reached a stable level at $14 \mathrm{kGy}$. So, it could be inferred that the absorbed dose of $14 \mathrm{kGy}$ could be selected as the saturated conversion dose to completely reduce $\mathrm{Pt}^{4+}$ with the concentration of $1 \mathrm{mM}$ to $\mathrm{Pt}^{0}$. The mechanism of PtNPs formation from $\mathrm{Pt}^{4+}$ ion solution by the gamma Co-60 ray irradiation was described in detail by Choi et al. [6] and Cele et al. [18]. Briefly, $\mathrm{Pt}^{4+}$ ions were reduced to $\mathrm{Pt}^{0}$ mainly by hydrated electrons $\left(e_{\mathrm{aq}}^{-}\right)$, hydrogen radicals $(\mathrm{H})$, and hydroxyl radicals $(\mathrm{OH})$. For $\mathrm{OH}$ radicals, they were converted into secondary radicals by hydrogen abstraction from free radical scavengers (alcohol, polysaccharides, etc.) for reducing $\mathrm{Pt}^{4+}$ ions.

The effect of CTS concentration was studied for the initial $\mathrm{Pt}^{4+}$ concentration of $1 \mathrm{mM}$. Figure 2 indicates that there was almost no difference among UV-Vis spectra of $\mathrm{PtNPs} / \mathrm{CTS}$ samples with different CTS concentrations. In addition, the size of the radiolytic synthesized PtNPs in Figure 3 was virtually not affected by the CTS concentrations from 0.5 to $2.0 \%$.

The synthesized PtNPs have a rather small size and narrow size distribution in the range of $1.4-1.6 \mathrm{~nm}$ (Figure 3). The size of resultant PtNPs with the concentration of $1 \mathrm{mM}$ in this study was smaller than the size of 4.4 and 


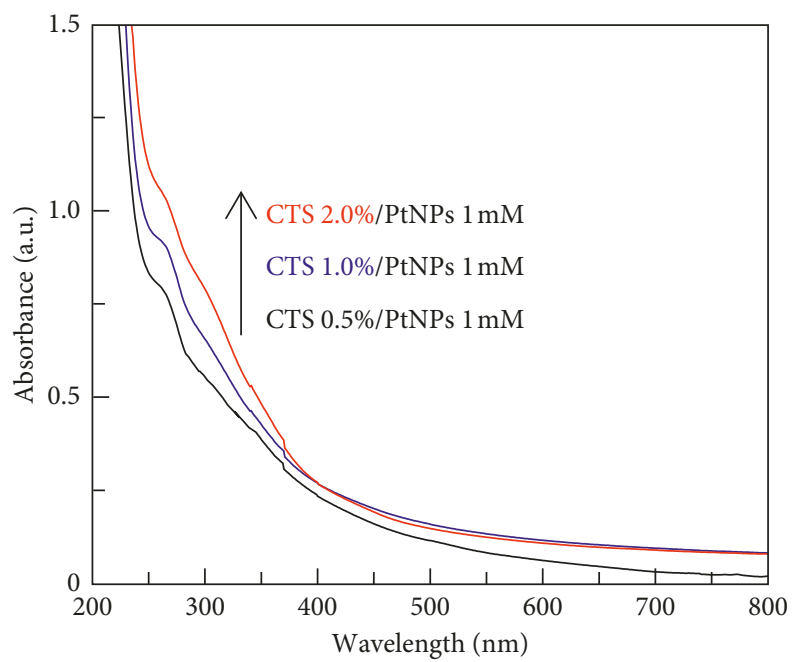

FIgURe 2: UV-Vis spectra of PtNPs/CTS solutions with different CTS concentrations.
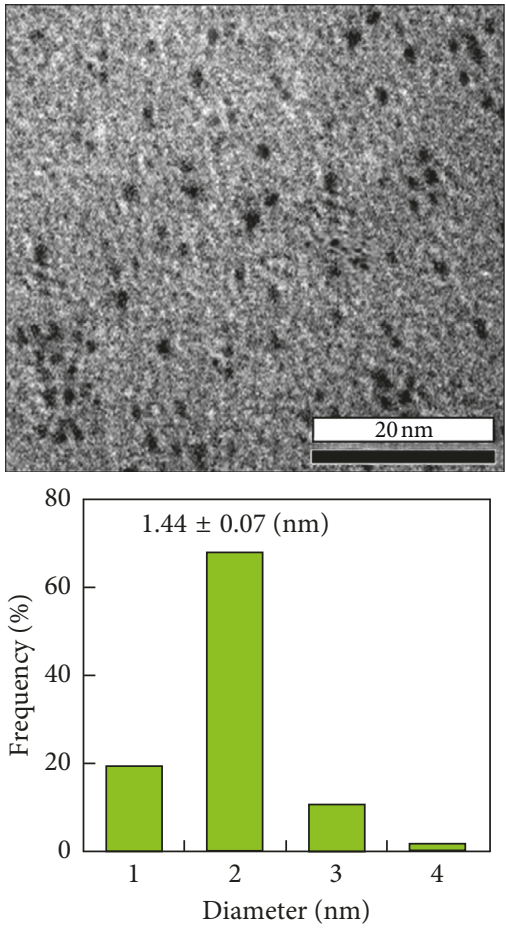

(a)
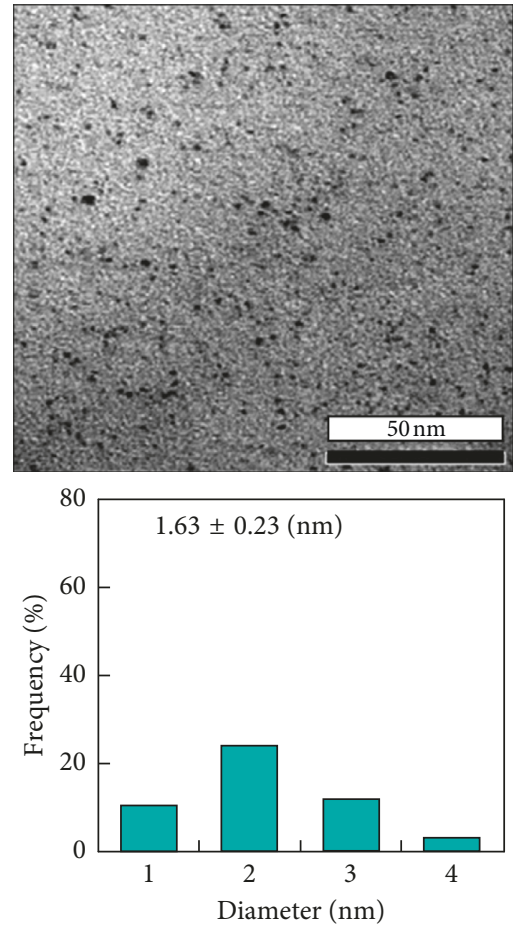

(b)
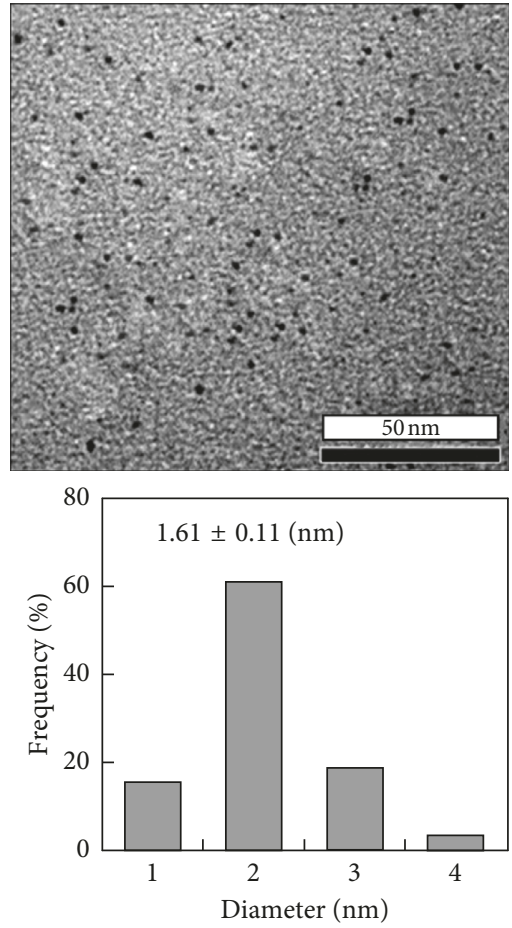

(c)

Figure 3: TEM images and size distribution of PtNPs from $1 \mathrm{mM}$ PtNPs in $0.5 \%$ CTS (a), $1.0 \%$ CTS (b), and $2.0 \%$ CTS (c).

$7.4 \mathrm{~nm}$ for the PtNPs concentrations of 3.4 and $10 \mathrm{mM}$, respectively $[17,18]$. Furthermore, the size of the synthesized PtNPs $(1.4-1.6 \mathrm{~nm})$ was also smaller than that of the PtNPs $(3.4 \mathrm{~nm})$ that were synthesized by the chemical method using $\mathrm{NaBH}_{4}$ as a reducing agent with the same initial concentration of $1 \mathrm{mM} \mathrm{Pt}^{4+}$ and $1 \%$ CTS as a stabilizer [5]. Moreover, the study on the synthesis of Ag and $\mathrm{Au}$ nanoparticles by the gamma Co-60 ray irradiation method using CTS as a stabilizer revealed that the size of $\mathrm{Ag}$ and $\mathrm{Au}$ nanoparticles was 4.6 and $9.8 \mathrm{~nm}$ for $1 \mathrm{mM} \mathrm{Ag}^{+}$and $\mathrm{Au}^{3+}$ ions, respectively $[16,20]$. Thus, it is obvious that the size of PtNPs synthesized by the gamma Co-60 ray irradiation method is smaller than that of $\mathrm{Ag}$ and $\mathrm{Au}$ nanoparticles at the same initial concentration of metal ions. The reason for this phenomenon is still not clear. Furthermore, it is interesting to note that, according to Choi et al. [6], the size of PtNPs which was synthesized by the gamma Co-60 ray irradiation using polyvinyl pyrrolidone as a stabilizer and the $\mathrm{Pt}^{4+}$ ions concentration of $0.2 \mathrm{mM}$ could not be predicted from TEM images due to the morphology of particles was not clear. Therefore, further study of the effect of stabilizers on the morphology and size of PtNPs 


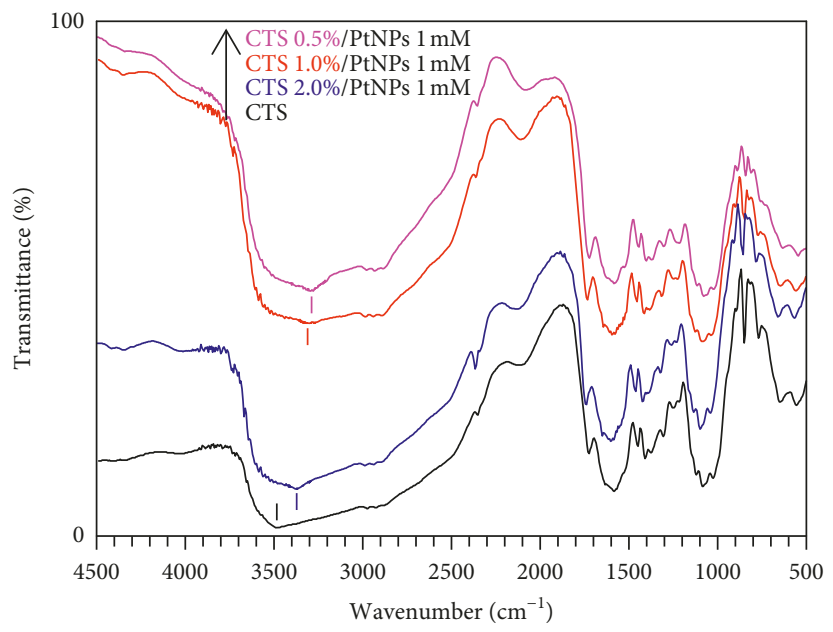

Figure 4: FT-IR spectra of CTS and PtNPs/CTS samples with different CTS concentrations.

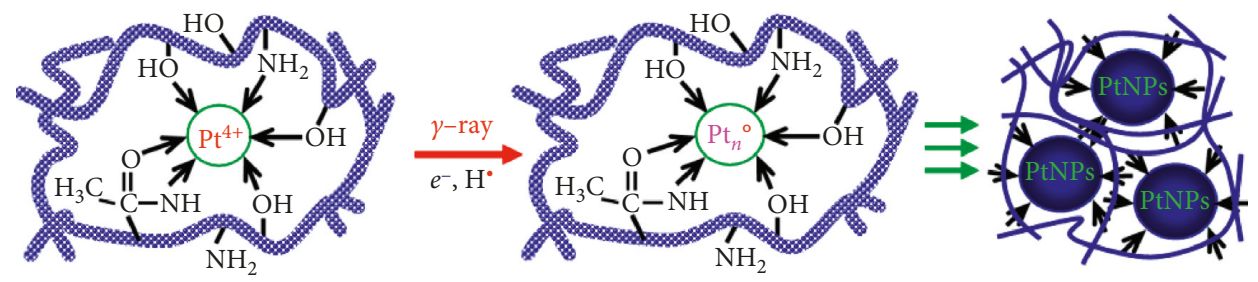

Figure 5: Schematic diagram of chitosan capped PtNPs synthesized by the gamma Co-60 ray irradiation.

synthesized by the gamma-ray irradiation method should be carried out.

The FT-IR spectra of CTS and PtNPs/CTS are shown in Figure 4. The FT-IR spectrum of the CTS possesses the main characteristic peaks that appear at $3448 \mathrm{~cm}^{-1}$ for $\mathrm{O}-\mathrm{H}$ and N-H stretch; $2937 \mathrm{~cm}^{-1}$ and $2981 \mathrm{~cm}^{-1}$ for C-H stretch [21]; $1641 \mathrm{~cm}^{-1}$ for $\mathrm{C}=\mathrm{O}$ stretching vibration in amide $\mathrm{I}$; $1591 \mathrm{~cm}^{-1}$ for $\mathrm{N}-\mathrm{H}$ deformation vibration in amide II; $1413 \mathrm{~cm}^{-1}$ and $1380 \mathrm{~cm}^{-1}$ for acetamide stretching and $\mathrm{C}-\mathrm{CH}_{3}$ deformation vibration of amide groups; $1126 \mathrm{~cm}^{-1}$ for antisymmetric stretch $\mathrm{C}-\mathrm{O}-\mathrm{C}$ and $\mathrm{C}-\mathrm{N}$ stretch; and $1089 \mathrm{~cm}^{-1}$ and $1031 \mathrm{~cm}^{-1}$ for skeletal vibration of C-O stretch [21, 22]. The FT-IR spectra of PtNPs/CTS samples with different concentrations of CTS in Figure 4 are almost similar to that of CTS. However, the main difference was that the peak at $3448 \mathrm{~cm}^{-1}$ of CTS shifted to lower wavenumbers, particularly at 3382,3269 , and $3236 \mathrm{~cm}^{-1}$ for PtNPs/CTS samples with CTS concentration of $2.0,1.0$, and $0.5 \%$, respectively. Thus, on the basis of the FT-IR spectra of PtNPs/ CTS in Figure 4, it presumed that the interaction between CTS molecules and PtNPs has occurred. The obtained FT-IR spectra in this study were also consistent with that in the study of Geng et al. [21] using chitosan as a stabilizer for the synthesis of Fe nanoparticles.

Chitosan, hyaluronan, and alginate with oxygen-rich structures lead to binding them to metal nanoparticles tightly via steric and electrostatic interactions [23]. On the basis of the stabilizing interaction of polysaccharides to metal nanoparticles, a schematic diagram of chitosan capped PtNPs which were synthesized using the gamma Co-60 ray irradiation was proposed as in Figure 5. Furthermore, the spherical PtNPs with the small size (1-2 nm) were favorably synthesized using the gamma Co-60 ray irradiation. And this method has been considered as a green production method with favorable large-scale production [24, 25].

\section{Conclusions}

The gamma Co-60 ray irradiation has been as an effective method for synthesizing PtNPs dispersed in the CTS solution. The saturated conversion dose $\left(\mathrm{Pt}^{4+} \longrightarrow \mathrm{Pt}^{0}\right)$ for the initial $\mathrm{Pt}^{4+}$ concentration of $1 \mathrm{mM}$ was found out to be $\sim 14 \mathrm{kGy}$. The resultant PtNPs with the small size (1.4$1.6 \mathrm{~nm}$ ) could be potentially applied in catalyst materials and other purposes of applications as well.

\section{Data Availability}

The experimental and analytical data used to support the findings of this study are included within the article.

\section{Conflicts of Interest}

The authors declare that they have no conflicts of interest.

\section{Acknowledgments}

This research was funded by the Vietnam National Foundation for Science and Technology (NAFOSTED) under Grant no. "104.06-2017.311." The authors would like to thank 
VINAGAMMA Center, VINATOM, for providing gamma Co-60 irradiation and favorable experimental conditions.

\section{References}

[1] J.-J. Du, C. Chen, Y.-L. Gan, R.-H. Zhang, C.-Y. Yang, and X.-W. Zhou, "Facile one-pot hydrothermal synthesis of Pt nanoparticles and their electrocatalytic performance," International Journal of Hydrogen Energy, vol. 39, no. 31, pp. 17634-17637, 2014.

[2] H. Huang, P. Hu, H. Huang, J. Chen, X. Ye, and D. Y. C. Leung, "Highly dispersed and active supported Pt nanoparticles for gaseous formaldehyde oxidation: influence of particle size," Chemical Engineering Journal, vol. 252, pp. 320-326, 2014.

[3] W. Yang, Y. Ma, J. Tang, and X. Yang, "“Green synthesis” of monodisperse Pt nanoparticles and their catalytic properties," Colloids and Surfaces A: Physicochemical and Engineering Aspects, vol. 302, no. 1-3, pp. 628-633, 2007.

[4] S. Remita, M. Mostafavi, and M. O. Delcourt, "Bimetallic Ag.Pt and Au.Pt aggregates synthesized by radiolysis," $R a-$ diation Physics and Chemistry, vol. 47, no. 2, pp. 275-279, 1996.

[5] M. J. Laudenslager, J. D. Schiffman, and C. L. Schauer, "Carboxymethyl chitosan as a matrix material for platinum, gold, and silver nanoparticles," Biomacromolecules, vol. 9, no. 10, pp. 2682-2685, 2008.

[6] S.-H. Choi, Y.-P. Zhang, A. Gopalan, K.-P. Lee, and H.-D. Kang, "Preparation of catalytically efficient precious metallic colloids by $\gamma$-irradiation and characterization," Colloids and Surfaces A: Physicochemical and Engineering Aspects, vol. 256, no. 2-3, pp. 165-170, 2005.

[7] D. Rao, Q. Sheng, and J. Zheng, "Preparation of flower-like Pt nanoparticles decorated chitosan-grafted graphene oxide and its electrocatalysis of hydrazine," Sensors and Actuators B: Chemical, vol. 236, pp. 192-200, 2016.

[8] P. L. Suryawanshi, S. P. Gumfekar, P. R. Kumar, B. B. Kale, and S. H. Sonawane, "Synthesis of ultra-small platinum nanoparticles in a continuous flow microreactor," Colloid and Interface Science Communications, vol. 13, pp. 6-9, 2016.

[9] D. Tongsakul, K. Wongravee, C. Thammacharoen, and S. Ekgasit, "Enhancement of the reduction efficiency of soluble starch for platinum nanoparticles synthesis," Carbohydrate Research, vol. 357, pp. 90-97, 2012.

[10] L. Wu, S. Shafii, M. R. Nordin, K. Y. Liew, and J. Li, "Structure determination of chitosan-stabilized $\mathrm{Pt}$ and Pd based bimetallic nanoparticles by X-ray photoelectron spectroscopy and transmission electron microscopy," Materials Chemistry and Physics, vol. 137, no. 2, pp. 493-498, 2012.

[11] S. Dehdashtian, M. B. Gholivand, M. Shamsipur, and S. Kariminia, "Construction of a sensitive and selective sensor for morphine using chitosan coated $\mathrm{Fe}_{2} \mathrm{O}_{3}$ magnetic nanoparticle as a modifier," Materials Science and Engineering: $C$, vol. 58, pp. 53-59, 2016.

[12] Y. Ma, X. Wu, and G. Zhang, "Core-shell Ag@Pt nanoparticles supported on sepiolite nanofibers for the catalytic reduction of nitrophenols in water: enhanced catalytic performance and DFT study," Applied Catalysis B: Environmental, vol. 205, pp. 262-270, 2017.

[13] M. Zhao, N. Zhong, and Y. Ji, "Ultra-stable colloidal porous Pt-Au-Ag nanoparticles," Materials Letters, vol. 191, pp. 3841, 2017.

[14] A. Thirumurugan, P. Aswitha, C. Kiruthika, S. Nagarajan, and A. N. Christy, "Green synthesis of platinum nanoparticles using Azadirachta indica-an eco-friendly approach," Materials Letters, vol. 170, pp. 175-178, 2016.

[15] ASTM International, "Standard practice for use of a dichromate dosimetry system," in Standards on Dosimetry for Radiation Processing, ISO/ASTM 51401-2003(E), pp. 69-74, ASTM International, West Conshohocken, PA, USA, 2004.

[16] D. V. Phu, V. T. K. Lang, N. T. Kim Lan et al., "Synthesis and antimicrobial effects of colloidal silver nanoparticles in chitosan by $\gamma$-irradiation," Journal of Experimental Nanoscience, vol. 5, no. 2, pp. 169-179, 2010.

[17] E. Gharibshahi, E. Saion, A. Ashraf, and L. Gharibshahi, "Sizecontrolled and optical properties of platinum nanoparticles by gamma radiolytic synthesis," Applied Radiation and Isotopes, vol. 130, pp. 211-217, 2017.

[18] T. Cele, M. Maaza, and A. Gibaud, "Synthesis of platinum nanoparticles by gamma radiolysis," MRS Advances, vol. 3, no. 42-43, pp. 2537-2557, 2018.

[19] K. D. N. Vo, C. Kowandy, L. Dupont, X. Coqueret, and N. Q. Hien, "Radiation synthesis of chitosan stabilized gold nanoparticles comparison between $e^{-}$beam and $\gamma$ irradiation," Radiation Physics and Chemistry, vol. 94, pp. 84-87, 2014.

[20] N. D. Nguyen, V. P. Dang, A. Q. Le, and Q. H. Nguyen, "Electron beam $/ \gamma$-ray irradiation synthesis of gold nanoparticles and investigation of antioxidant activity," Advances in Natural Sciences: Nanoscience and Nanotechnology, vol. 5, no. 4, article 045002, 2014.

[21] B. Geng, Z. Jin, T. Li, and X. Qi, "Preparation of chitosanstabilized $\mathrm{Fe}^{0}$ nanoparticles for removal of hexavalent chromium in water," Science of The Total Environment, vol. 407, no. 18, pp. 4994-5000, 2009.

[22] J. Yang, J.-H. Yu, J. Rudi Strickler, W.-J. Chang, and S. Gunasekaran, "Nickel nanoparticle-chitosan-reduced graphene oxide-modified screen-printed electrodes for enzymefree glucose sensing in portable microfluidic devices," Biosensors and Bioelectronics, vol. 47, pp. 530-538, 2013.

[23] L. Huang, M. Zhai, J. Peng, L. Xu, J. Li, and G. Wei, "Synthesis, size control and fluorescence studies of gold nanoparticles in carboxymethylated chitosan aqueous solutions," Journal of Colloid and Interface Science, vol. 316, no. 2, pp. 398-404, 2007.

[24] M. Chandra and P. K. Das, "Green routes to noble metal nanoparticle synthesis," International Journal of Green Nanotechnology: Physics and Chemistry, vol. 1, no. 1, pp. P10-P25, 2009.

[25] N. Q. Hien, D. V. Phu, N. N. Duy, and L. A. Quoc, "Radiation synthesis and characterization of hyaluronan capped gold nanoparticles," Carbohydrate Polymers, vol. 89, no. 2, pp. 537-541, 2012. 


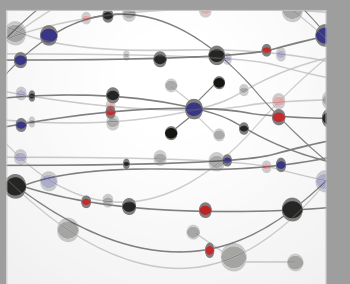

The Scientific World Journal
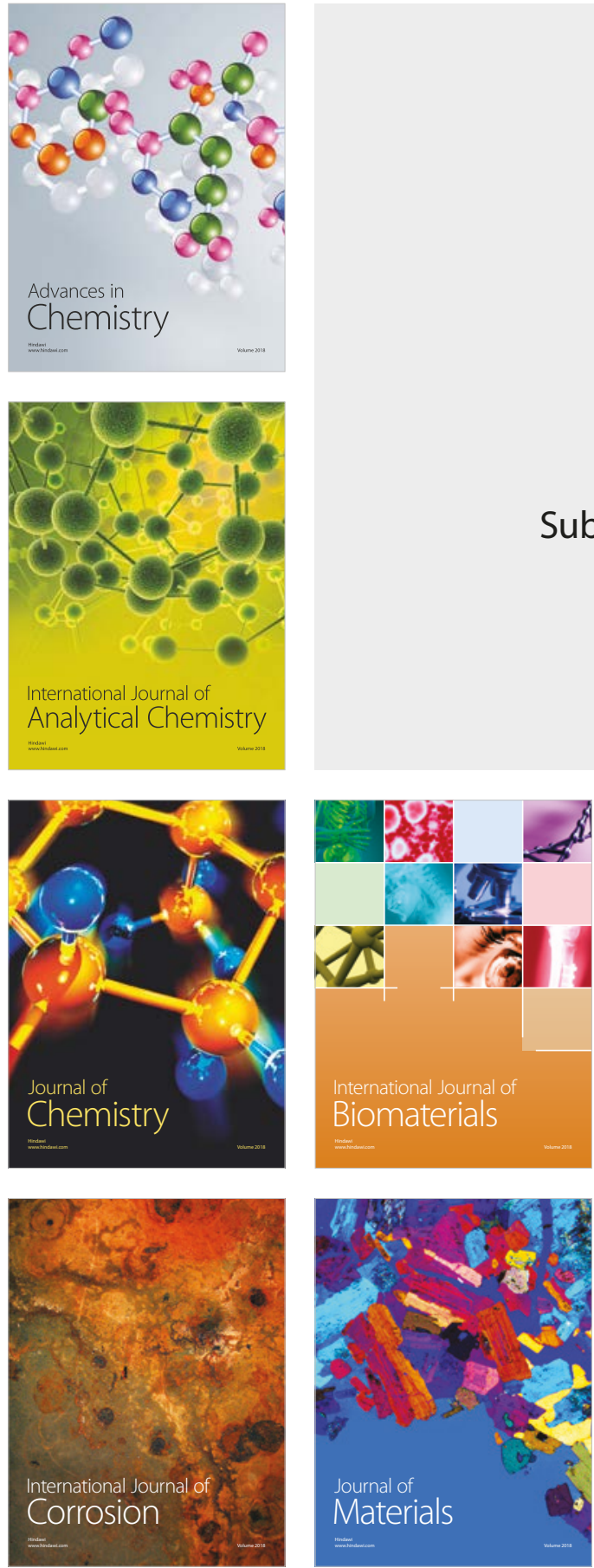

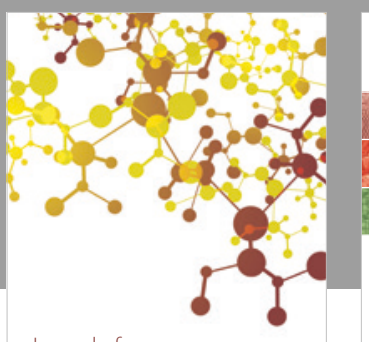

Journal of

Applied Chemistry
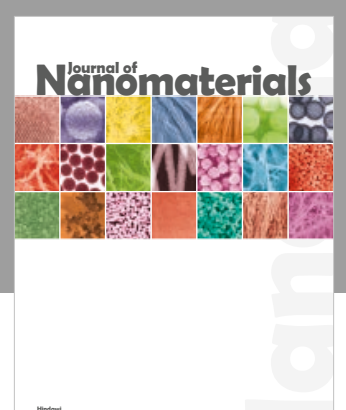

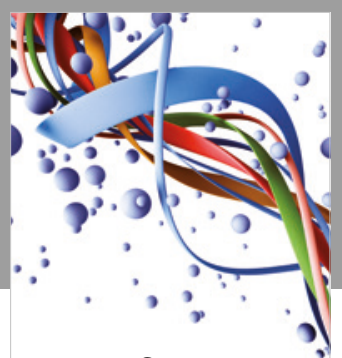

Scientifica

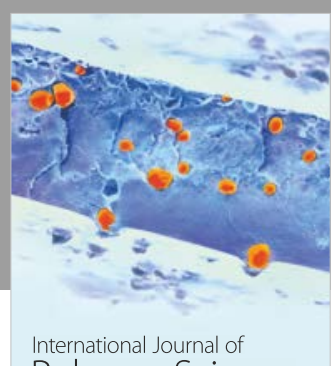

Polymer Science

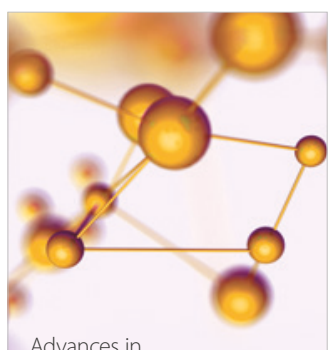

Physical Chemistry
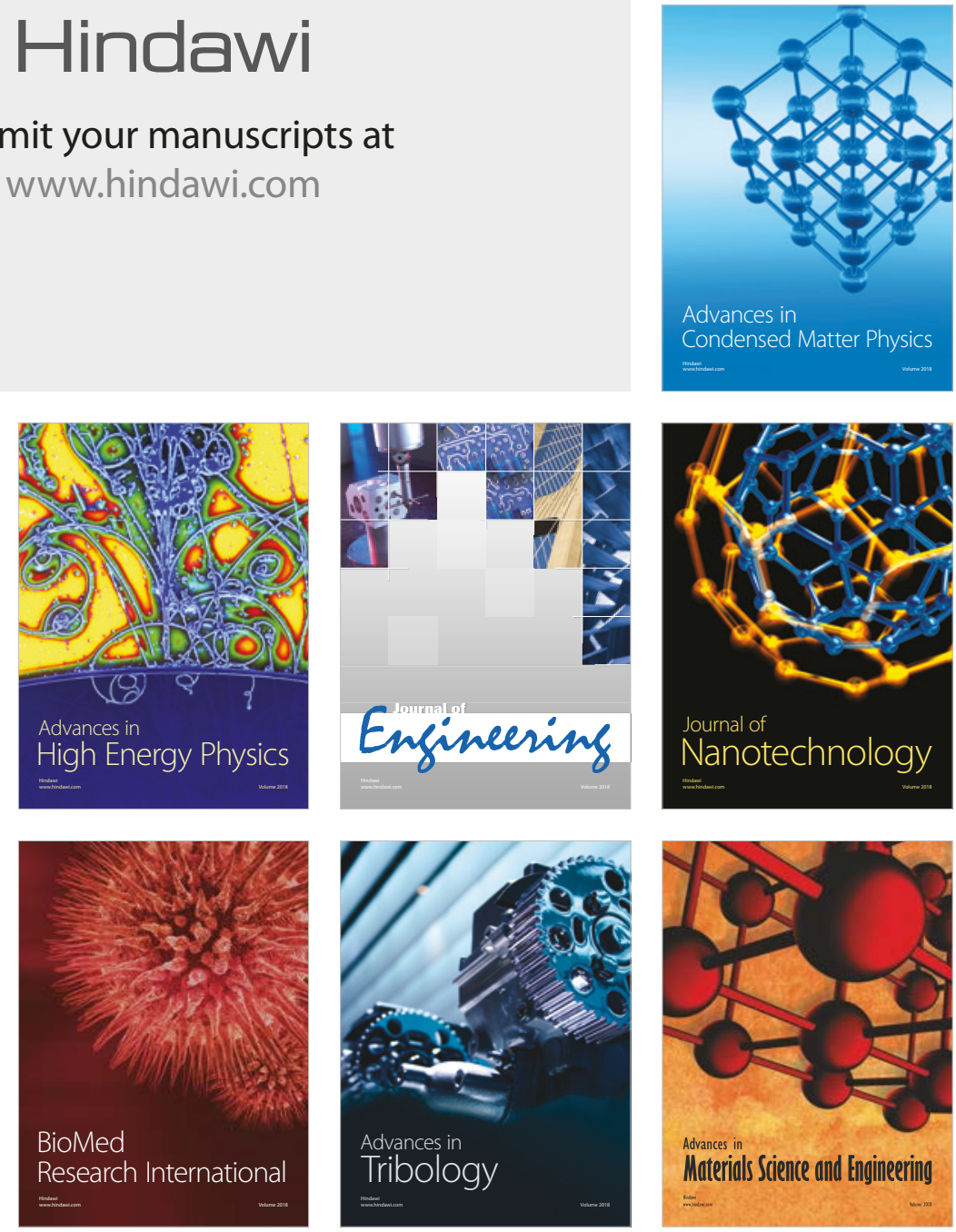